\title{
Value Frameworks for the Patient-Provider Interaction: A Comparison of the ASCO Value Framework Versus NCCN Evidence Blocks in Determining Value in Oncology
}

\author{
Bijal Shah-Manek, BPharm, PhD; Joseph S. Galanto, PharmD Candidate;
}

Huong Nguyen, PharmD, MPH Candidate; and Robert Ignoffo, PharmD, FASHP, FCSHP

\begin{abstract}
BACKGROUND: To address the rising concern about oncology drug costs, the American Society of Clinical Oncology (ASCO) and the National Comprehensive Cancer Network (NCCN) recently developed unique tools to help providers and patients make informed decisions about the value of an anticancer regimen. The ASCO Value Framework (AVF) allows users to generate a net health benefit (NHB) score along with drug acquisition costs for oncology regimens that have been compared in a prospective randomized clinical trial. In contrast, the NCCN Evidence Blocks (NEB) derives ratings from an expert panel assessment in the categories of efficacy, safety, quality and consistency of evidence, and affordability.
\end{abstract}

OBJECTIVE: To compare the results of the AVF and NEB by applying each tool to the same clinical scenarios.

METHODS: We evaluated 2 regimens using the AVF and NEB scores: (1) enzalutamide for treatment of metastatic castration-resistant prostate cancer and (2) nivolumab versus docetaxel in treatment of advanced squamous and nonsquamous non-small cell lung cancer (NSCLC).

RESULTS: Enzalutamide generated a total NHB score of 44.8 (range $0-180$ ) for use before chemotherapy and 70.8 for use after chemotherapy with a monthly cost of $\$ 8,495$ in the AVF. The NEB scored enzalutamide 4 (very effective) for efficacy, 4 (occasionally toxic) for safety, and 2 (expensive) for affordability in the no visceral metastases block. It scored 3 (moderately effective) for efficacy, 4 for safety, and 2 for affordability in the visceral metastases block. Nivolumab in advanced nonsquamous NSCLC scored 36.0 and 73.2 in advanced squamous NSCLC, with a monthly cost of $\$ 7,010$ in the AVF. The NEB gave nivolumab a score of 4 for efficacy and safety and 1 (very expensive) for affordability in the NEB in advanced nonsquamous and advanced squamous NSCLC.

CONCLUSIONS: The AVF and NEB are novel tools that take different approaches in assessing the value of an oncology treatment regimen. From this study, it is clear that the findings generated by these tools are distinct. The AVF provides a summary score for treatments across all clinical benefit and toxicity categories, whereas the NEB provides component scores for treatment efficacy, safety, quality and consistency of evidence, and affordability. Both tools are novel and come with their own challenges.

J Manag Care Spec Pharm. 2017;23(6-a):S13-S20

Copyright $\odot 2017$, Academy of Managed Care Pharmacy. All rights reserved.

\section{What is already known about this subject}

The ASCO Value Framework (AVF) compares 2 regimens that have been studied in a prospective randomized clinical trial by generating a net health benefit score and comparing the drug acquisition cost of each regimen.

The National Comprehensive Cancer Network Evidence Blocks (NEB) represents average values from an expert panel in a matrix assessing treatment efficacy, safety, quality and consistency of evidence, and affordability; scores are based on clinical trials and expert panel consensus and range from 1 to 5, with 1 being least favorable and 5 being most favorable.

\section{What this study adds}

Although both frameworks are useful, there is considerable variability in the value output generated by the tools because of the substantial differences in assessment criteria and scoring methodology. In its current form, the AVF does not add to the clinical decisionmaking process because of the difficulty of scoring and variability in results, especially with regard to the toxicity component.

While NEB scores were fairly consistent for the drugs in this study, they are subjective, and the rating process is not transparent, especially with regards to the affordability ratings.

$\mathrm{B}$ y 2020, cancer care costs are projected to reach approximately $\$ 158$ billion, up from $\$ 125$ billion in 2010. ${ }^{1}$ The increasing prevalence of cancer coupled with the rising cost of new drugs and technologies has brought increased scrutiny to the cost and value of treatments in oncology. With new cancer drugs averaging $\$ 10,000$ a month, many patients are facing tough financial choices between paying for treatment or paying for the mortgage. ${ }^{2}$ Physicians are faced with decisions to determine if a potential treatment is not only clinically beneficial but also avoids putting the patient in financial harm.

To address this rising concern about oncology drug costs, several organizations have developed frameworks to assess the value of an oncology regimen. These frameworks include the American Society of Clinical Oncology (ASCO) Value Framework, the European Society for Clinical Oncology (ESMO) Value Framework, the National Comprehensive Cancer 
Network (NCCN) Evidence Blocks, the Institute for Clinical and Economic Review (ICER) reports, and the Memorial Sloan Kettering Cancer Center's DrugAbacus. ${ }^{3}$ Although these frameworks are useful for discussing the value of oncology regimens, they vary considerably in their definition of value and in their key characteristics, including target audience, methods to measure cost and benefit, evidence sources, and value output.

Of the oncology value frameworks mentioned above, the ASCO Value Framework (AVF) and the NCCN Evidence Blocks (NEB) have the same intended target audience and purposethey aim to assist providers and patients to make informed decisions about the value of an oncology regimen. ${ }^{1,4}$ To do this, the AVF compares 2 different cancer treatment regimens from a head-to-head randomized clinical trial (RCT). The AVF framework integrates scores for efficacy-overall survival, progression-free survival, disease-free survival, or response rate-as well as safety (toxicity) in generating the net health benefit (NHB) score, potentially resulting in a maximum score of $180 .{ }^{2}$ Both the overall NHB score and drug cost are then used to assess the value of the proposed treatment.

In contrast, the NEB is generated from an expert panel that rates 5 components-treatment efficacy, safety, quality and consistency of evidence, and affordability using a standardized scale from 1 to 5 , where 1 is the least favorable and 5 is the most favorable. Scores are then plotted onto a 5-by-5 matrix to produce a visual plot of the panel members' responses. ${ }^{5}$ However, no overall score is generated to compare treatments as is done in the AVF tool.

AVF and NEB are designed for providers to use in their conversations with patients to help inform individual treatment decisions. Although both tools are intended to assist at the patient-provider level, the frameworks differ greatly in their methodology and inputs. One of the key differences between the tools is in the type of evidence and the scoring system used in each tool. The AVF is scored primarily based on prospective head-to-head RCTs with a maximum NHB score of 180. In contrast, NEB's scoring is based on a standardized scale from 1 to 5 and incorporates data from meta-analyses, randomized control trials, case reports, and clinical experience and incorporates panel members' subjective assessment of the treatment. NCCN also allows for manufacturers to submit evidence, and panel members may also use nonpublished data as part of their decision making. ${ }^{6}$

The AVF awards bonus points for tail-of-the-curve survival benefit, palliation of cancer symptoms, quality of life, and treatment-free interval; the NEB does not award bonus points. The 2 frameworks also differ vastly in their assessment of costs-AVF looks only at the direct cost of the drug, including drug acquisition cost and the patient's out-of-pocket cost. In contrast, NCCN looks at affordability of the overall treatment, taking into account other costs of therapy such as hospitalization, supportive care, and administration.
To measure safety, AVF measures clinically relevant adverse events, whereas the NCCN panel assesses safety endpoints based on expert opinion. Finally, NCCN assesses the quality and consistency of evidence, whereas ASCO does not include a methodology to assess the quality or consistency of evidence used in its value framework.

Some recent pilot studies have attempted to compare the value frameworks and assess their reliability and validity; however, findings are inconsistent. ${ }^{3,7-9}$ In the study by Wilson et al. (2017), ${ }^{3}$ the authors report that the AVF has low interrater reliability, especially with regard to scoring the toxicity component of the NHB score. On the other hand, the study by Bentley et al. (2017) showed that the AVF had high interrater reliability and that the AVF and NEB showed convergent validity. ${ }^{9}$ In this study, we sought to compare the results of the AVF and NEB by applying each tool to the same clinical scenarios.

\section{Methods}

In this study, the revised AVF and NEB were compared using 2 common cancers-prostate cancer and non-small cell lung cancer (NSCLC). We compared enzalutamide versus placebo for metastatic castration-resistant prostate cancer (MCRPC) and nivolumab versus docetaxel for advanced nonsquamous and squamous NSCLC. Enzalutamide was chosen, as it has been used as an example in the AVF and also has published Evidence Blocks rated by an expert panel. Nivolumab versus docetaxel was chosen as the second treatment regimen for comparison because these drugs treat a common disease state, lung cancer.

We searched PubMed to identify head-to-head phase 3, RCTs that compared enzalutamide versus placebo in treatment of MCRPC and nivolumab versus docetaxel in the treatment of NSCLC. NHB scores for enzalutamide versus placebo were calculated from published trials evaluating its use before and after chemotherapy, whereas scores for nivolumab versus docetaxel were calculated using 2 recently published comparative trials in squamous and nonsquamous NSCLC. ${ }^{10-13}$

AVF scores were calculated by 2 fourth-year PharmD student authors (Galanto and Nguyen) and verified by a PhD-trained researcher and principal investigator with experience in oncology health outcomes research (Shah-Manek). Each scorer independently calculated the NHB score for each drug using the trials chosen for inclusion in this study. An Excel spreadsheet was used to calculate the scores for each component of the AVF and the overall NHB score. To validate our method of calculating the AVF scores, we also calculated the NHB scores for enzalutamide and compared them with those published as part of the AVF framework.

All studies chosen for calculation of AVF scores were phase 3 studies and were also used by the NCCN in writing the Guidelines for MCRPC and NSCLC. To compare NHB scores 
Value Frameworks for the Patient-Provider Interaction: A Comparison of the ASCO Value Framework Versus NCCN Evidence Blocks in Determining Value in Oncology

TABLE 1 ASCO Value Framework Calculations: Enzalutamide Versus Placebo

\begin{tabular}{|c|c|c|}
\hline & Metastatic Prostate Cancer After Chemotherapy & Metastatic Prostate Cancer Before Chemotherapy \\
\hline Clinical benefit score & 37 & 29 \\
\hline HR for death & 0.63 & 0.71 \\
\hline Clinical benefit calculation & $(1-0.63) \times 100 \times 1=37$ & $(1-0.71) \times 100 \times 1=29$ \\
\hline Toxicity score & -2.2 & -4.2 \\
\hline Enzalutamide & 15 & 32 \\
\hline Placebo & 13.5 & 26.5 \\
\hline Toxicity calculation & $(15 \div 13.5)-1=0.11 ; 0.11 \times-20=-2.2$ & $(32 \div 26.5)-1=0.21 ; 0.21 \times-20=-4.2$ \\
\hline Total bonus points & 36 & 20 \\
\hline Tail of the curve & 16 & 0 \\
\hline Palliation & 10 & 10 \\
\hline Treatment-free interval & 0 & 0 \\
\hline Quality of life & 10 & 10 \\
\hline Net health benefit (\%) & 70.8 & 44.8 \\
\hline Net health benefit calculation & $37-2.2+36=70.8$ & $29-4.2+20=44.8$ \\
\hline Drug acquisition cost (\$ per month) & 8,495 & 8,495 \\
\hline Cost per unit of benefit $(\$)$ & 119.98 & 189.62 \\
\hline Cost per unit of benefit calculation (\$) & $8,494.91 \div 70.8=119.98$ & $8,494.91 \div 44.8=189.62$ \\
\hline Percentage of NHB score & 39.3 & 24.9 \\
\hline Percentage of NHB score calculation & $70.8 \div 180=0.393 \times 100=39.3$ & $44.8 \div 180=0.249 \times 100=24.9$ \\
\hline \multicolumn{3}{|c|}{$\begin{array}{l}\text { Note: Clinical benefit, toxicity, and NHB scores were calculated by following the instructions outlined by the ASCO Value Framework version } 2 \text {, using } 2 \text { separate clini- } \\
\text { cal trials that compared enzalutamide versus placebo in metastatic prostate cancer before chemotherapy and metastatic prostate cancer after chemotherapy. } 10,11 \text { Costs } \\
\text { are taken directly from the ASCO Value Framework article and are based on average sales price as of October } 2014 \text { for intravenous therapies and on information from } \\
\text { UnitedHealthcare for oral drugs. }{ }^{2} \text { Cost per unit of NHB was calculated by dividing the drug acquisition cost by the calculated NHB score. Percentage of NHB score was } \\
\text { calculated by dividing the calculated NHB score by the maximum score possible (180). } \\
\text { ASCO=American Society of Clinical Oncology; HR= hazard ratio; NHB= net health benefit. }\end{array}$} \\
\hline
\end{tabular}

for the same drug across trials, we calculated 2 additional metrics - the NHB percentage and the cost per unit of NHB. These additional calculations are not part of the AVF and were used by the investigators to normalize results for comparisons.

The monthly drug acquisition cost for enzalutamide was taken from the AVF, which was based on the average sales price as of October 2014 for intravenous therapies and data from United Healthcare for oral drugs. ${ }^{2}$ The monthly (4-week) costs for nivolumab were calculated using average wholesale price (AWP) as of June 2016 from Lexicomp and dosing for NSCLC, using the dose of $240 \mathrm{mg}$ (flat dose) once every 2 weeks. ${ }^{14} \mathrm{NEB}$ value scores for enzalutamide versus placebo and nivolumab versus docetaxel were developed by an expert panel of NCCN members and were used for comparison..$^{15,16}$

\section{Results}

\section{Enzalutamide: ASCO Value Framework}

Enzalutamide has been used in the treatment of MCRPC both before and after chemotherapy. The first clinical trial used in the AVF was a comparison of enzalutamide versus placebo in the treatment of MCRPC after chemotherapy. ${ }^{11}$ The clinical benefit score for enzalutamide was generated using hazard ratio (HR) for death (clinical benefit score 37). The toxicity score was calculated using only grade 3 or higher toxicities due to unclear reporting of lower-grade toxicities (toxicity score -2.2).
Tail-of-the-curve, palliation, and quality-of-life bonus points were awarded (36 additional points).

The final calculated NHB score for enzalutamide was 70.8; range 0-180). The monthly drug acquisition cost was $\$ 8,495$. Additional calculations for cost per unit of NHB and NHB percentage were also performed and were $\$ 120$ per unit of NHB and 39.3\% (71 of 180), respectively. A summary of the calculations is shown in Table 1 and graphically represented in Figure 1. Use of enzalutamide resulted in a 37\% reduction in risk of death and slightly more clinically relevant toxicity in comparison with placebo (15 vs. 13.5).

The second trial used for calculating enzalutamide scores was a phase 3 study comparing enzalutamide to placebo in metastatic prostate cancer before chemotherapy. ${ }^{10}$ The calculated NHB score from this study was 44.8 , which is $25 \%$ of the total NHB score. Assuming the same monthly drug acquisition cost of $\$ 8,495$, the calculated cost per unit of NHB would be $\$ 190$ per unit of NHB. Use of enzalutamide resulted in a 29\% reduction in risk of death and more clinically relevant toxicity in comparison with placebo (32 vs. 26.5). The calculations are detailed in Table 1.

\section{Enzalutamide: NCCN Evidence Blocks}

For the use of enzalutamide, the NEB treatment of MCRPC was different (Version 3.2016), depending on the presence or 


\section{FIGURE 1 ASCO Value Framework: Enzalutamide Versus Placebo for Treatment in Metastatic} Castration-Resistant Prostate Cancer After Chemotherapy

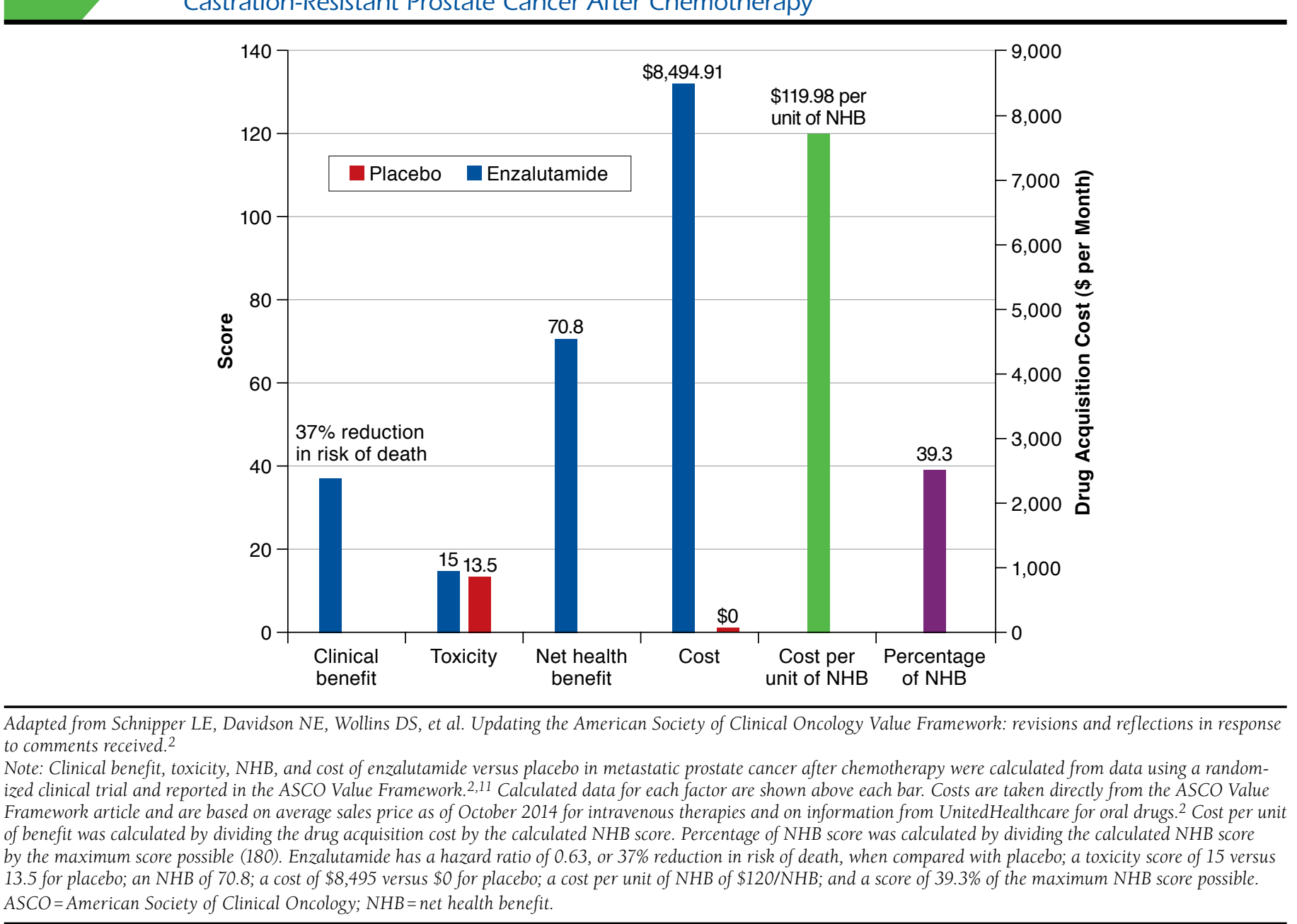

absence of visceral metastases, as shown in detail in Figure 2. ${ }^{16}$ For no visceral metastases, the NEB had a score of 4 (very effective) for efficacy, 4 (occasionally toxic) for safety, 4 (good) for quality, and 4 (mainly consistent) for consistency and 2 (expensive) for affordability. The NEB score for visceral metastases was similar to the NEB score for no visceral metastases, with the exception that efficacy was scored 3 (moderately effective).

\section{Nivolumab: ASCO Value Framework}

The clinical trial used in calculating the NHB score for nivolumab was a phase 3 study comparing nivolumab with docetaxel in advanced nonsquamous NSCLC. ${ }^{12}$ The calculated AVF clinical benefit score was 27 , using HR for death. The final toxicity score for nivolumab was 9.0, due to a subtraction of 5 points for treatment-related endocrine toxicities that were not expected to be resolved. The final calculated NHB score for nivolumab was 36.0 (range 1-180) and a monthly drug acquisition cost of $\$ 7,010$ based on AWP. The cost per unit of NHB and NHB percentage were calculated as $\$ 195$ per unit of NHB and $20.0 \%$ (36 of 180) of the total NHB. A summary of the calculations is shown in Table 2. As can be seen in Figure 3, use of nivolumab resulted in a $27 \%$ reduction in risk of death and was associated with less clinically relevant toxicity (as defined by the AVF) than docetaxel (17.5 vs. 57.5).

The phase 3 study performed by Brahmer et al. (2015) was also used in calculating the NHB score for nivolumab versus docetaxel in advanced squamous NSCLC. ${ }^{13}$ Calculated NHB score in this trial was 73.2. Using the previously mentioned drug acquisition cost of nivolumab $(\$ 7,010)$, the cost per unit of NHB was $\$ 96$. The NHB percentage was $40.7 \%$ (73.2 of 180) and had 20 tail-of-the-curve bonus points awarded for having a greater portion of patients alive at 2 times the median overall 


\section{FIGURE 2 NCCN Evidence Blocks: Enzalutamide for Treatment in Metastatic} Castration-Resistant Prostate Cancer (Version 3.2016)16

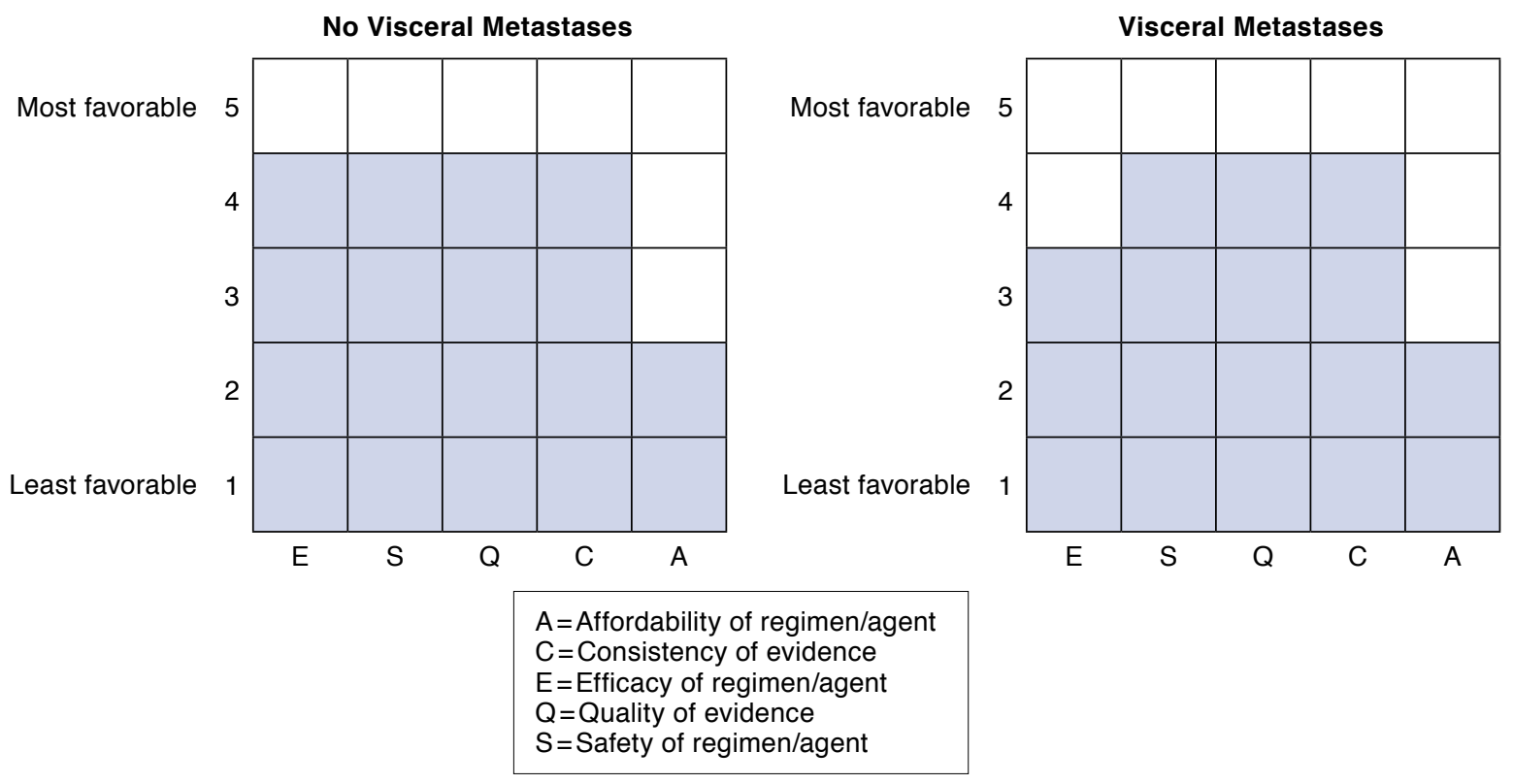

Adapted with permission from the NCCN Clinical Practice Guidelines in Oncology (NCCN Guidelines) with NCCN Evidence Blocks for Prostate Cancer V3.2016. (C) 2016 National Comprehensive Cancer Network, Inc. All rights reserved. The NCCN Guidelines and illustrations herein may not be reproduced in any form for any purpose without the express written permission of NCCN. To view the most recent and complete version of the NCCN Guidelines, go online to NCCN.org. The NCCN Guidelines are a work in progress that may be refined as often as new significant data becomes available. NCCN makes no warranties of any kind whatsoever regarding their content, use or application and disclaims any responsibility for their application or use in any way.

NCCN = National Comprehensive Cancer Network

survival. A summary of the calculations is shown in Table 2. Based on the data from this trial, nivolumab resulted in a $41 \%$ reduction in risk of death and was associated with substantially less clinically relevant toxicity in comparison with docetaxel (23.5 vs. 59.5).

\section{Nivolumab: NCCN Evidence Blocks}

The results from the NEB show that nivolumab had a better efficacy and safety profile compared with docetaxel (4 vs. 3 for docetaxel on both measures). The quality of evidence was higher for docetaxel (5 vs. 4 for nivolumab); both drugs were scored the same on consistency of evidence. However, there was a large difference in affordability (4 vs. 1 for nivolumab). The NEB for the use of nivolumab in advanced nonsquamous NSCLC was identical to the NEB in nonsquamous NSCLC, but docetaxel differed in that the quality of the evidence was lower (dropping from 5 to 4 ).

\section{Discussion}

Our results showed that the AVF scores are highly dependent on the clinical trial used as the data source and are quite variable. The NHB scores for enzalutamide ranged from 45 to
71 in 2 randomized controlled trial studies versus placebo, indicating a benefit to the drug, but to what extent is unclear. The NEB determined by the NCCN expert panel also showed a benefit of enzalutamide in metastatic prostate cancer, but the scores for both scenarios were nearly identical. In contrast, the results for the nivolumab studies showed that the AVF scores (36 vs. 73.2 ) were more widely spread and could be attributed to the different stages of NSCLC, but both showed benefit for the drug. The significance of these results is not truly known because the meaning of an AVF score is uncertain at this time.

In its current form, the AVF does not add to the clinical decision-making process due to the difficulty of scoring and variability in results, especially with regard to the toxicity component. These results are consistent with the research of Wilson et al. who found that the interrater reliability (kappa coefficient) of the NHB score across 11 oncology practitioners was only 0.11 (slight reliability), with the toxicity component having the lowest reliability (kappa 0.06). ${ }^{3}$ In our study also, toxicity scores showed the widest variability, with enzalutamide toxicity scores ranging from -5.8 to -2.2 and nivolumab toxicity scores ranging from 9 to 12.2 . Wilson et al. also showed that the NHB scores lacked variability throughout the full range 
Value Frameworks for the Patient-Provider Interaction: A Comparison of the ASCO Value Framework Versus NCCN Evidence Blocks in Determining Value in Oncology

TABLE 2 ASCO Value Framework Calculations: Nivolumab Versus Docetaxel

\begin{tabular}{|c|c|c|}
\hline & Advanced Nonsquamous NSCLC & Advanced Squamous-Cell NSCLC \\
\hline Clinical benefit score & 27 & 41 \\
\hline HR for death & 0.73 & 0.59 \\
\hline Clinical benefit calculation & $(1-0.73) \times 100 \times 1=27$ & $(1-0.59) \times 100 \times 1=41$ \\
\hline Toxicity score & 9.0 & 12.2 \\
\hline Nivolumab & 17.5 & 23.5 \\
\hline Docetaxel & 57.5 & 59.5 \\
\hline Toxicity calculation & $1-(17.5 \div 57.5)=0.70 ; 0.70 \times 20=14 ; 14-5=9$ & $1-(23.5 \div 59.5)=0.61 ; 0.61 \times 20=12.2$ \\
\hline Total bonus points & 0 & 20 \\
\hline Tail of the curve & 0 & 20 \\
\hline Palliation & 0 & 0 \\
\hline Treatment-free interval & 0 & 0 \\
\hline Quality of life & 0 & 0 \\
\hline Net health benefit (\%) & 36.0 & 73.2 \\
\hline Net health benefit calculation & $27+9.0+0=36$ & $41+12.2+20=73.2$ \\
\hline Drug acquisition cost (\$ per month) & 7,010 & 7,010 \\
\hline Cost per unit of benefit (\$) & 194.72 & 95.76 \\
\hline Cost per unit of benefit calculation (\$) & $7,009.86 \div 36=194.72$ & $7,009.86 \div 73.2=95.76$ \\
\hline$\%$ of NHB score & 20.0 & 40.7 \\
\hline$\%$ of NHB score calculation & $36 \div 180=0.200 \times 100=20.0$ & $73.2 \div 180=0.407 \times 100=40.7$ \\
\hline \multicolumn{3}{|c|}{$\begin{array}{l}\text { Note: Clinical benefit, toxicity, and NHB scores were calculated by following the instructions outlined by the ASCO Value Framework, using } 2 \text { separate clinical trials that } \\
\text { compared nivolumab versus docetaxel in advanced nonsquamous NSCLC and in advanced squamous-cell NSCLC.12,13 Monthly ( } 4 \text {-week) costs for nivolumab were calcu- } \\
\text { lated using average wholesale price as of June } 2016 \text { from Lexicomp and dosing for NSCLC using the dose of } 240 \text { mg (flat dose) once every } 2 \text { weeks. }{ }^{14} \text { Cost per unit of NHB } \\
\text { was calculated by dividing the drug acquisition cost by the calculated NHB score. Percentage of NHB score was calculated by dividing the calculated NHB score by the } \\
\text { maximum score possible (180). } \\
\text { ASCO=American Society of Clinical Oncology; HR=hazard ratio; NHB=net health benefit; NSCLC=non-small cell lung cancer. }\end{array}$} \\
\hline
\end{tabular}

of scores, with scores in the study ranging only through the bottom third of the possible scores ( -3.4 to 66 , possible scores up to 180). Additionally, they noted that NHB scores in the upper 25\%-50\% might not be achievable, given the structure of the scoring system.

To reduce variability in NHB scores, Wilson et al. suggest that the AVF needs stricter guidelines to specify the comparator used for each drug. ${ }^{3}$ Given the low reliability, the lack of variability in scores, and the difficulty of scoring the toxicity component of the AVF, they concluded that the framework was not ready for use in clinical practice. Our findings generally support the conclusions of this study.

It is also noteworthy to mention that the NHB scores cannot be compared across trials. Therefore, an NHB generated from 1 clinical trial cannot be compared to an NHB score generated from a different clinical trial due to potential differences in each study's design. This may be considered a serious limitation of the framework, as it does not allow inclusion of data from multiple trials. Furthermore, results generated from similar trials for the same drug are not comparable, as seen in this study.

In a report that surveyed 50 oncologists and 55 payers about AVF, both groups agreed that the inability to compare treatments across clinical trials was a major limitation of the framework. ${ }^{17}$ Additionally, the heavy reliance on data from clinical trials, the lack of consideration of data from obser- vational studies or nonrandomized studies, and the inability to grade the quality/quantity of information are other major limitations of the framework. By excluding real-world evidence studies, the AVF may provide an incomplete picture of the drug's overall benefit.

To compare AVF scores across trials, we computed the cost per unit of NHB and the overall NHB percentage. For enzalutamide, the cost per unit of NHB varied from $\$ 120$ to $\$ 190$ per unit of NHB, whereas the NHB percentage varied from $25 \%$ to $39 \%$. For nivolumab, the cost per unit of NHB varied from $\$ 96$ to $\$ 195$ per unit, whereas the NHB percentage varied from $20 \%$ to $41 \%$. As noted earlier, the AVF scores showed variability when looking at NHB scores, cost per unit of NHB, and toxicity/clinical benefit scores. NEB scores for the drugs evaluated in this study showed very little variability, if any. Scores for nivolumab in both squamous and nonsquamous NSCLC were identical in all 5 categories. Scores for enzalutamide in both scenarios were identical for 4 categories, whereas there was a 1-point difference in efficacy scores.

While NEB scores were fairly consistent for the drugs in this study, they are subjective, as they are based on expert panel members' knowledge of the data and their clinical experience. Furthermore, the process of generating the NEB scores is not fully transparent, especially as it relates to the ratings of affordability. Panel members are asked to rate the affordability of a treatment regimen using their knowledge of 


\section{FIGURE 3 ASCO Value Framework: Nivolumab Versus Docetaxel for Treatment in Advanced Nonsquamous} Non-Small Cell Lung Cancer

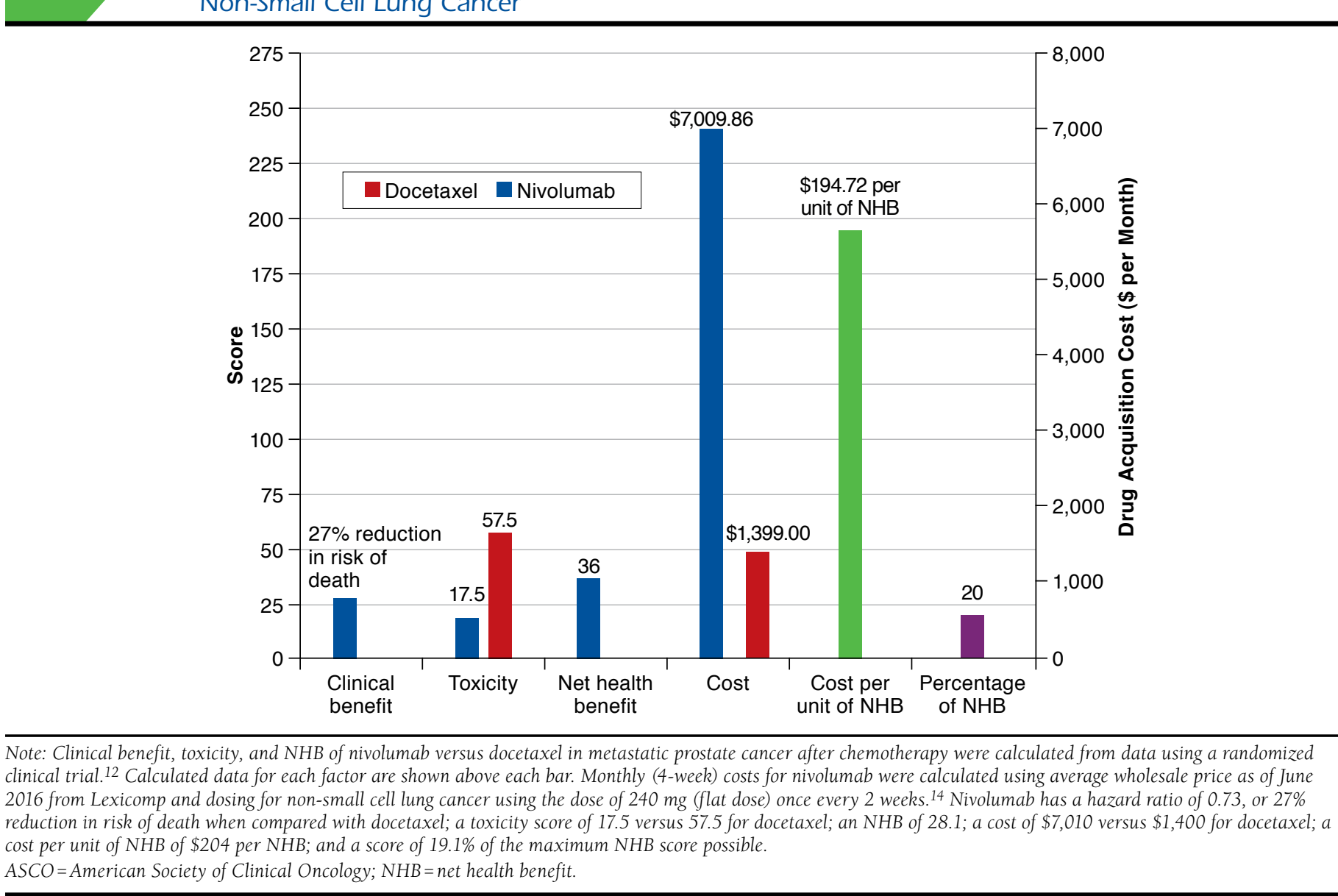

the overall cost of the regimen, including the cost of the drug, administration costs, supportive care costs, and costs to manage adverse events, including hospitalization. No information is provided to panel members to rate the affordability of an oncology regimen (personal communication, NCCN staff).

Given the opaque nature of drug pricing in the United States, are oncologists accurately rating affordability? What elements factor into the affordability rating of a drug, and are these ratings aligned with actual drug costs? Future research should attempt to determine if oncologists' ratings of affordability as part of the NEB are reliable and valid. In addition, research is needed to determine if affordability ratings are affected by practice characteristics, patient characteristics, or demographics of the oncologist panel member. Until then, it is not certain if the NEB scores can be considered reliable and valid.

Further, both tools do not allow for personal value assessment. Value is highly individualized and is different for patients and providers. Some patients may deem that toxicity is more important than survival benefits or vice versa. To adjust for this, each tool should have a way to customize the assessment to each patient's own personal value system. Frequency of dosing, quality of life, cost due to lost time and productivity, and caregiver burden are examples of factors that can be valuable to a patient and may affect treatment choice.

As an example, a patient with metastatic breast cancer, where cure is not possible, may value quality of life over length of life. Another patient with early-stage breast cancer, where cure is the goal, may have a higher threshold for toxicity in order to maximize survival. In this scenario, value is determined by each patient's unique life circumstances and is likely to affect patient preference for treatment. Future iterations of the tool should consider incorporating the ability to customize and weight the components of the tool to allow for a personalized value assessment.

\section{Limitations}

The data produced for this study are limited in that only 2 oncology regimens were assessed in both frameworks. 
Further, given the disparity in the rating scales and evaluation methods, no statistical analysis was conducted on the results. Finally, to compare AVF scores across trials, we computed the cost per unit of NHB and the overall NHB percentage; however, this method has not been validated.

\section{Conclusions}

The increased cost of cancer care has brought forth 2 different tools to assess value in oncology drugs and to assist the patient and provider in making informed treatment choices. From this study, it is clear that the output generated by these tools is quite distinct. The AVF provides a summary score for treatments across all clinical benefit and toxicity categories, whereas the NEB provides component scores for treatment efficacy, safety, quality and consistency of evidence, and affordability. Both tools are novel and come with their own challenges.

\section{Authors}

BIJAL SHAH-MANEK, BPharm, PhD, Touro University California College of Pharmacy, Vallejo, California, and Ipsos Healthcare, San Francisco, California. JOSEPH S. GALANTO, PharmD Candidate; HUONG NGUYEN, PharmD, MPH Candidate; and ROBERT IGNOFFO, PharmD, FASHP, FCSHP, Touro University California College of Pharmacy, Vallejo, California.

AUTHOR CORRESPONDENCE: Bijal Shah-Manek, BPharm, PhD, Associate Professor, Social, Behavioral and Administrative Science, Touro University California College of Pharmacy, 1310 Club Dr., Vallejo, CA 94592. Tel.: 650.452.3777; E-mail: Bijal.Shah@tu.edu.

\section{DISCLOSURES}

No outside funding supported this study. Shah-Manek is also employed by Ipsos Healthcare, a consulting firm. The authors have no conflicting interests to report.

Study concept and design were contributed by Shah-Manek and Ignoffo. Galanto and Nguyen collected the data, and data interpretation was performed by all the authors. All the authors contributed to writing the manuscript, which was revised primarily by Shah-Manek, along with Galanto, Nguyen, and Ignoffo.

This research was previously presented as a poster and podium presentation at the Academy of Managed Care Pharmacy Nexus 2016 held October 3-6 in National Harbor, Maryland.

\section{REFERENCES}

1. Schnipper LE, Davidson NE, Wollins DS, et al. American Society of Clinical Oncology statement: a conceptual framework to assess the value of cancer treatment options. J Clin Oncol. 2015;33(23):2563-77.
2. Schnipper LE, Davidson NE, Wollins DS, et al. Updating the American Society of Clinical Oncology Value Framework: revisions and reflections in response to comments received. J Clin Oncol. 2016;34(24):2925-34.

3. Wilson L, Lin T, Wang L, et al. Evaluation of the ASCO Value Framework for anticancer drugs at an academic medical center. J Manag Care Spec Pharm. 2017;23(2):163-69. Available at: http://www.jmcp.org/doi/pdf/10.18553/ jmcp.2017.23.2.163

4. Gross CP, Emanuel EJ. A call for value in cancer research. JAMA Oncol. 2016;2(1):11-12

5. NCCN Clinical Practice Guidelines in Oncology (NCCN Guidelines) with NCCN Evidence Blocks. 2016. Available at: https://www.nccn.org/evidenceblocks/default.aspx. Accessed April 11, 2017.

6. Westrich K. Current landscape: value assessment frameworks. National Pharmaceutical Council. Updated June 2016. Available at: http://www. npcnow.org/system/files/research/download/npc-current-landscape-valueassessment-frameworks-final_0.pdf. Accessed April 26, 2017.

7. Cohen JT, Anderson JE, Neumann PJ. Three sets of case studies suggest logic and consistency challenges with value frameworks. Value Health. 2017;20(2):193-99.

8. Mandelblatt JS, Ramsey SD, Lieu TA, Phelps CE. Evaluating frameworks that provide value measures for health care interventions. Value Health. 2017;20(2):185-92.

9. Bentley TG, Cohen JT, Elkin EB, et al. Validity and reliability of value assessment frameworks for new cancer drugs. Value Health. 2017;20(2):200-05.

10. Beer TM, Armstrong AJ, Rathkopf DE, et al. Enzalutamide in metastatic prostate cancer before chemotherapy. N Engl J Med. 2014;371(5):424-33.

11. Scher HI, Fizazi K, Saad F, et al. Increased survival with enzalutamide in prostate cancer after chemotherapy. N Engl J Med. 2012;367(13):1187-97.

12. Borghaei H, Paz-Ares L, Horn L, et al. Nivolumab versus docetaxel in advanced nonsquamous non-small-cell lung cancer. N Engl J Med. 2015;373(17):1627-39.

13. Brahmer J, Reckamp KL, Baas P, et al. Nivolumab versus docetaxel in advanced squamous-cell non-small-cell lung cancer. N Engl J Med. 2015;373(2):123-35

14. Lexicomp Online. Clinical drug information. Nivolumab. Wolters Kluwer. December 9, 2016. Available at: http://www.wolterskluwercdi.com/ lexicomp-online/. Accessed April 11, 2017.

15. Referenced with permission from the NCCN Clinical Practice Guidelines in Oncology (NCCN Guidelines) for with NCCN Evidence Blocks for NonSmall Cell Lung Cancer Version 4.2016. (C) National Comprehensive Cancer Network, Inc. 2016. All rights reserved. Accessed April 11, 2017. To view the most recent and complete version of the guideline, go online to NCCN.org.

16. Referenced with permission from the NCCN Clinical Practice Guidelines in Oncology (NCCN Guidelines) for with NCCN Evidence Blocks for Prostate Cancer Version 3.2016. (C) National Comprehensive Cancer Network, Inc. 2016. All rights reserved. Accessed April 11, 2017. To view the most recent and complete version of the guideline, go online to NCCN.org.

17. Xcenda. Assessing value in oncology: models \& perspectives from payers and oncologists. Page 4. 2016. Available at: http://www.xcenda.com/ Documents/Assessing\%20Value\%20in\%200ncology\%20-\%20Models\%20 $\% 20$ Perspectives $\% 20$ from $\% 20$ Payers $\% 20$ and $\% 20$ Oncologists $\% 20$ FINAL\%20 for\%20print\%205-18-16.pdf. Accessed April 17, 2017. 